\title{
BMLA virtual annual conference and educational courses
}

6-7th May 2021

www.bmla.co.uk

Disclaimer: Abstracts published as submitted by submitter

INV01 Developments in semi-permanent make up: tips and advice for laser removal

\section{Mrs Rhiannon Smith ${ }^{1}$}

${ }^{T}$ Lynton Lasers Ltd, United Kingdom

Session 1A: Nursing and Therapist Session, May 6, 2021, 13:30 - 14:45

\section{Biography:}

Rhiannon has been working in the aesthetics industry for over 14 years and joined Lynton as a Laser Practitioner in 2010. She has been part of the Clinical Training team since 2015. As well as equipment training, Rhiannon also teaches the VTCT Level 4 and 5 Qualifications.

Dubbed by British Vogue as one of 2021's key beauty trends, cosmetic tattooing is an increasingly popular procedure, used to create long-lasting eye liner, lip liner, and brow definition. As this procedure grows in popularity, we are seeing more salons offering these time-saving treatments and a corresponding increase in the number of clients looking to have the ink removed.

In this presentation, we will look at some of the results that can be obtained when using lasers to remove semi-permanent make-up, alongside practical tips for treatment, and a discussion of some of the challenges encountered.

INV02 A review of patients treated with ablative lasers for skin malignancies

\section{Mrs Natalie Allen ${ }^{1}$}

${ }^{T}$ Bedford Hospital NHS Trust, United Kingdom

Session 1A: Nursing and Therapist Session, May 6, 2021, 13:30 - 14:45

\section{Biography:}

Natalie is a Skin Cancer/Laser Clinical Nurse Specialist at Bedford Hospital NHS Trust. She provides support to patients, carers, and colleagues and runs regular surveillance clinics for patients that have had a skin cancer diagnosis and a weekly laser clinic. Her role has influenced her interest in laser treatment as an option when managing and treating pre-cancerous skin lesions and some skin malignancies.

Although the gold standard treatment of skin cancer is surgery, which allows a histological diagnosis and can confirm the clearance of excision, there is a cohort of patients that cannot undergo surgery for various reasons, for example, comorbidities and extent of the disease or those who require palliative management. This is where ablative lasers are now playing a major role in treatment and disease control for these patients.
A short review of some cases where patients have had or continue to have benefit from $\mathrm{CO}_{2}$ laser treatment for skin malignancies (e.g., basal cell carcinomas, lentigo maligna, and cutaneous metastatic melanoma).

INV03 Managing laser safety and COVID-19

\author{
Mrs Lindsey Simcox ${ }^{1}$ \\ ${ }^{T}$ Aurora Health Physics Services Ltd, United Kingdom \\ Session 1A: Nursing and Therapist Session, May 6, 2021, 13:30 - 14:45
}

\section{Biography:}

Lindsey is a certificated Laser Protection Adviser at Aurora Health Physics. She has provided advice and training for medical laser users for over 10 years.

The COVID-19 pandemic has created a number of additional challenges for routine laser safety management.

This presentation aims to highlight the key areas where laser users should consider how their work may be affected going forward and will give practical advice to help ensure compliance with the relevant legislation and standards.

INV04 Rosacea: developments in recognising and treating the sub-types of this progressive, inflammatory vascular disorder

\section{Miss Kerry Belba ${ }^{1}$}

${ }^{\text {TLaser Skin Solutions Ltd }}$

Session 1A: Nursing and Therapist Session, May 6, 2021, 13:30 - 14:45

\section{Biography:}

Laser Practitioner, Laser Trainer, and Key Opinion Leader. Kerry is the owner of Laser Skin Solutions Bournemouth and was the first non-medical practitioner in the UK to obtain the BTEC Laser \& Light award in 2005. She has obtained the Level 4 CIBTAC and Level 5 VTCT laser qualifications, in addition to being a laser trainer. With 16 years' experience, she specialises in the aesthetic application of laser treatments with a particular interest in acne, acne scarring and rosacea. Kerry is the first aesthetic BMLA board member.

Treating rosacea with lasers and IPLs has been the gold standard treatment for over a decade. Recognising the sub-types of this, often debilitating, condition however is crucial to getting the best treatment outcomes.

In this presentation, we will be looking at what the different sub-types are, how they present, and how best to approach treatment. 
O01 Laser hair removal for inflammatory medical conditions: pilonidal sinus disease

\author{
Miss Liliana Marza ${ }^{1}$ \\ ${ }^{T}$ LaserSkinTreatment, www.laserskintreatment.co.uk, ${ }^{2}$ Skin 55 \\ Dermatology, London, UK, ${ }^{3}$ The London Clinic, London, UK \\ Session 1A: Nursing and Therapist Session, May 6, 2021, 13:30 - 14:45
}

\section{Biography:}

Liliana is a Laser Nurse Specialist with her own private practice at No 55 Harley Street, a large dermatological clinic in Central London. She has a scientific and peri-operative care background with more than 20 years' experience working with multiple different lasers. She specialises in treating medical conditions such as pilonidal disease, vascular, and complex dermatological conditions.

Liliana publishes in peer-reviewed journals and contributed to the NICE Guidelines on Pilonidal Sinus Disease management. She is a member of the International Pilonidal Society and a Specialist Advisor to the Care Quality Committee (CQC) role in which she inspects NHS hospitals and private practices.

Laser hair removal (LHR) is the most frequently used aesthetic treatment behind "injectables" (botox \& fillers). Less often reported are the benefits of LHR in medical conditions either as an adjuvant or definite treatment. This presentation discusses LHR in pilonidal sinus disease (PSD).

PSD is a disabling condition of young men and women caused by the penetration of hairs into the skin of the natal cleft leading to acute infection and chronic sinus formation. Primary treatment may need to be incision and drainage and in the later stages by excision of sinuses; however there is a very high recurrence rate due to re-accummulation of hairs in the wound. A course of LHR with meticulous wound care significantly reduces recurrence and transforms patients' quality of life. The presentation will explain how LHR is applied with clinical examples and a review of the literature.

INV07 Far UVC inactivation of SARS-CoV-2: a safe approach to combat the spread of COVID

Dr Paul O'Mahoney ${ }^{1}$

${ }^{1}$ University of Dundee, United Kingdom

Session 2A: Scientific Developments, May 6, 2021, 13:30 - 14:45

\section{Biography:}

Dr Paul O'Mahoney is a Post-Doctoral Research Assistant working within the Photobiology Unit at Ninewells hospital. He obtained his PhD in Physics in 2015 and his BEng (Hons) in Mechanical Engineering in 2011, both from the University of Dundee.

His research is focused on the applications of physics in Photodynamic Therapy and Photodiagnosis and the translation of technologies into healthcare.

The COVID-19 pandemic has brought about an accelerated interest in disinfection technologies to help mitigate the spread of viruses, in this case SARS-CoV-2. One such technology is ultraviolet-C (UVC) irradiation, which is known to inactivate similar viruses efficiently. UVC inactivation is by no means a new technology, with its use dating back at least to the late 19 th century.

A hurdle in the implementation of UVC technologies for inactivation is the translation of laboratory research to practical use situations, and how the inactivation outcomes might differ between the two. The safety of UVC sources is also of paramount importance - practical implementation of UVC irradiation must adhere to the published exposure limit values.

This talk presents a brief overview of the inactivation of SARS-CoV-2, and other viruses, by FarUVC irradiation. The talk also discusses implementation of FarUVC technologies and what should reasonably be expected from FarUVC in the 'real world'.
O03 Developing a predictive model to determine effectiveness of a laser using an artificial neural network

Mr Kavish Maheshwari ${ }^{1}$, Ms Kerry Muggeson ${ }^{1}$, Ms Roxana Chiru ${ }^{1}$, Prof Sandip Hindocha

${ }^{1}$ Bedford hospital NHS Trust, Bedford, United Kingdom

Session 2A: Scientific Developments, May 6, 2021, 13:30 - 14:45

\section{Biography:}

I am a speciality doctor in plastic and reconstructive surgery at Bedford hospital and an honorary research fellow at the University of Bedfordshire. I did my graduation and masters in general surgery from Delhi and plastic surgery training from Calcutta, India.

Artificial intelligence is making its inroads into clinical practice. The way machine learning works is through algorithms which identify specific features and compares them with the diagnostic information provided. This is the basis of artificial neural networks (ANN). These can safely be called a diagnostic tool of the future. A future that is already here. Image classification has been at the forefront of ANN, which are currently being developed for use in dermatology. There are apps which are used to determine the likelihood of a pigmented lesion being malignant, which is an example of using these systems to improve diagnostic accuracy.

We propose to develop an ANN, which would be specific to benign conditions that are treated using lasers. Tattoos, vascular lesions, hair removal, and pigmented lesions are commonly treated with lasers, but there is still a doubt before starting treatment, how likely is the lesion or condition going to respond to a particular laser.

Developing an ANN will improve treatment efficacy for lasers in predicting outcome, and they can be used as prognostic and educational tools. One can simply create applications for phones which can click a picture of a condition and determine the likelihood that particular condition has of responding to a particular wavelength/laser.

We are collaborating with the University of Bedfordshire for development of such a tool, which would be useful for everyone dealing with lasers.

O04 Understanding the physical mechanisms involved in laser irradiation of tattoos

\section{Mr Michael Murphy ${ }^{1}$}

${ }^{T}$ DermaLase Research Unit, Glasgow, Scotland Session 2A: Scientific Developments, May 6, 2021, 13:30 - 14:45

\section{Biography:}

Mike Murphy has been working in the medical laser field since 1986 when he joined in the clinical research group which established scar-free removal of tattoos by $Q$-switched ruby laser. He currently researches both the theory and practical applications of laser/IPL tissue interactions.

Laser tattoo removal has been around since the early 1980s. Since that time both nanosecond and picosecond lasers have become standard tools. However, the physical processes which occur during, and immediately after, irradiation of tattoo particles is still not well understood.

To properly understand these processes, a number of important issues must be examined including the physical structure of tattoos, the absorption (and scattering) characteristics of inks, the temperature-time history during and after irradiation, reflection of incident laser energy due to steam bubble formation, the heat conduction properties of ink immersed in tissue water and subsequent physiological repair mechanisms and their timings.

In particular, the pulse duration of the light energy is important since intra-pulse thermal conduction can be significant, leading to lower peak temperatures for longer pulsewidths. This can determine how much incident energy is absorbed by the ink particles and how much is reflected back into the dermis due to steam formation, thereby determining the final outcome. 
Detailed calculations reveal that 'fragmentation' of ink particles is highly unlikely. Nor does plasma formation occur at the typical laser powers used. Results from a series of computer simulations will be presented.

O05 The role of a hospital LPA during COVID

\section{Dr Vincent Pelling ${ }^{1}$}

${ }^{T}$ University Hospitals Sussex NHS Trust, Brighton, United Kingdom

Session 2A: Scientific Developments, May 6, 2021, 13:30 - 14:45

\section{Biography:}

Vincent Pelling is an RPA2000 accredited LPA providing advice to NHS trusts and external organisations. He is also Chair of IPEM ultrasound and non-ionising radiation special interest group.

COVID and lockdown disrupted and changed hospital services in the space of a few weeks. Laser facilities were closed, moved and in cases newly commissioned. The traditional role of a hospital LPA was no longer practicable. Face-to-face visits and audits were no longer possible. Audits, commissioning, training and investigations all had to be conducted remotely.

This talk covers the practical experience of providing laser protection advice across several hospitals: the use of available on-line learning, the use of in-house training (for LPSs, users, and general staff), remote commissioning and auditing of rooms and equipment.

The success of remote laser protection advice will be assessed as restrictions lift and visits resume.

Several questions are considered. As restrictions lift does the LPA role revert to its former format? What is the optimal relationship between LPA and LPS? Can training be changed to meet target audiences better and more effectively? How does what has happened reflect on the awareness of the role of the LPA across a trust? How involved should an LPA be in the local running of lasers?

O06 Plume control in medical and cosmetic laser clinics: a practical guide

\section{Dr Godfrey Town ${ }^{1}$}

${ }^{T}$ GCG Healthcare L $t$, United Kingdom

Session 2A: Scientific Developments, May 6, 2021, 13:30 - 14:45

\section{Biography:}

Godfrey was awarded his Ph.D. in cutaneous light-based therapy at the University of Wales Trinity Saint David, Swansea and holds a Cardiff University Law School Expert Witness Certificate focussing on laser injury cases. He is a Senior Research Fellow at Alborg University Hospital, Dermatology Department, Aalborg, Denmark. He is a UK Registered Clinical Technologist specialising in the comparative measurement of intense pulsed light (IPL) and laser devices and has published over 25 peer-reviewed scientific and clinical papers. He also holds an RPA2000 Certificate of Competence in Non-Ionising Radiation, is a Fellow of the American Society for Laser in Medicine and Surgery (ASLMS) and a member of the European Society for Laser Dermatology (ESLD) and the BMLA. He sits on IEC, Cenelec and ANSI laser safety standards committees and chairs the Home Use Devices Safety Group. A former private laser and IPL clinic owner-manager; his current UK laser safety consultancy supports 250 private medical practices, clinics and spas using more than 300 laser and IPL devices.

Enhanced infection control and ventilation, driven by the COVID-19 pandemic, has required a review of associated policies and procedures particularly regarding Personal Protective Equipment (PPE). Accompanying this is a growing awareness by regulatory agencies and users of energy-based devices that produce airborne particulates and noxious plumes and the associated hazards to practitioners working routinely with such devices in the treatment of patients/clients.

It is the legal duty of the medical/cosmetic clinic to undertake appropriate risk assessments to identify such hazards, determine who might be harmed and how, evaluate the risk level and decide upon precautions and to keep these assessments under regular review.

This talk reviews the risk assessment process for plume control and examines recent publications focussing on the potential hazards linked with laser and intense pulsed light (IPL) hair reduction treatments. Practical recommendations are made to minimise plume in the medical/cosmetic workplace and current options for UV disinfection and face mask selection are given.

INV05 The MHRA's regulation of medical devices post 2021 and MHRA advice on lasers and IPLs

\section{Mr Andrew Marsden ${ }^{1}$}

${ }^{T}$ Medicines and Healthcare Regulatory Agencyodern Humanities Research Association, United Kingdom

Session 1B: Regulation and Training in the COVID-19 Era, May 6, 2021, 15:00 - 16:00

\section{Biography:}

Andrew Marsden is a Senior Medical Device Specialist within the MHRA's Device Safety and Surveillance Group. Andy investigates adverse incidents involving a wide range of medical devices within the categories of Assistive Technology, Medical Electrical and Ophthalmics.

This session looks at the recent changes to the UK regulation of medical devices and the legislation involved, highlights some of the changes afoot at MHRA mainly due to these changes and also explores why and how our advice document on medical lasers and IPL may soon change: https:/bit.ly/ 3fczAtk

The Medicines and Medical Devices bill transfers the regulatory decision-making formerly carried out by the European Commission to the UK Medicines and Healthcare products Regulatory Agency. This bill received Royal Assent on 11 February 2021, and the enacted primary legislation is now called the UK Medicines and Medical Devices Act 2021. After the debate given to patient safety during the bill's readings, especially the review by Baroness Cumberlege's team, "First do no Harm" (the report of the Independent Medicines and Medical Devices Safety Review), which was published last year, patient safety has been elevated to Part One of the Act. Medical devices are Part 4 of the Act, which effectively allows the secretary of state to amend or supplement the Medical Devices Regulations 2002 (SI 2002/618), the UK's statutory instrument for medical device regulation.

O02 Lasers in general dental practice - selection, safety and status

\section{Mr Graham Hart ${ }^{1}$}

${ }^{T}$ YourRPA, Morecambe, United Kingdom

Session 1B: Regulation and Training in the COVID-19 Era, May 6, 2021, 15:00 - 16:00

\section{Biography:}

Graham Hart worked as a medical physicist at Bradford Royal Infirmary for 30 years before leaving in 2004 to set up his own Radiation \& Laser Protection Adviser consultancy - YourRPA (www.YourRPA.co.uk).

\section{Selection:}

A wide range of lasers are now used in dental practice, including low power diodes for low level laser therapy, class 4 diode lasers for soft tissue treatments, and Er:YAG, Nd:YAG and Er:Cr:YSGG lasers for hard tissue treatments.

\section{Safety:}

The use of lasers in general dental practice presents a number of issues, since (naturally) the treatments all take place in the mouth and the dentist is likely to be closer to the laser sources than in many other medical and aesthetic laser applications. The design of dental surgeries may also give 
rise to other laser safety issues due to multiple reflective surfaces in and around the treatment area.

\section{Status:}

The status of an LPA appointment has been a source of some confusion, as although recommended in MHRA and BDA Guidance Documents, and a requirement for dental practices in both Scotland and Wales, there has been some confusion regarding the need for the appointment of an LPA in England, since dental lasers are not separately registerable there. However, CQC's 'Dental Mythbuster \#26' updated in October 2018 states that an LPA and LPS must be appointed, and the practices must have a laser safety management system in place. Subsequent correspondence with CQC has confirmed that the appointment of an LPA is a requirement for practices. All three topics will be discussed, highlighting the key role of the LPA, LPS and other professionals involved to provide a safe environment for the use of lasers in dentistry.

INV08 PDT: how I do it and what's new

Dr Colin Morton ${ }^{1}$

${ }^{7}$ NHS Forth Valley, United Kingdom

Session 2B: Photodynamic Therapy (PDT), May 6, 2021, 15:00 - 16:00

\section{Biography:}

Dr Colin A. Morton, Consultant Dermatologist, NHS Forth Valley, Stirling and Honorary Clinical Associate Professor.

Dermatology advisor to Chief Medical Officer (Scotland).

$M D$ thesis was in PDT for skin cancer and has published widely on topic. He is co-author of UK and International guidelines for best practice in skin cancer.

Photodynamic Therapy for use in Dermatology has evolved since its initial description of use in skin cancer over 25 years ago. Topical PDT is widely approved for actinic keratoses (AK), Bowen's disease, and certain basal cell carcinomas, both as lesional or field therapy, and has the potential to delay/reduce the development of new lesions.

There is now choice in photosensitisers and light sources. Three agents are currently licensed for use in Europe, methyl aminolaevulinate (MAL), nanoemulsion of 5-ALA and a patch containing 5-ALA. Conventional PDT involves occlusion of the photosensitizer for 3-4 hours, then illuminating typically by a narrowband red LED light. Daylight is increasingly used as the light source in treating $\mathrm{AK}$ and for field cancerization, with application of either nanoemulsion ALA or MAL for 0.5 hour, followed by exposure to daylight for 2 hours, with no inferiority of efficacy to red light PDT, but with greatly reduced pain. There is also an option for patients to wear a portable LED device. A novel light-emitting, fabric-based laser diode device has recently been shown to be as effective as conventional PDT in clearing AK but with minimal pain, with MAL applied for 30 minutes then fabric device is applied and switched on after 30 minutes, remaining on for 150 minutes.

There is an emerging literature on enhancing conventional PDT protocols or combined PDT with another treatment to increase response rates. Many additional indications have been evaluated, including photo-rejuvenation and inflammatory and infective dermatoses.

0O7 Head and neck PDT — where next?

\section{Mr Colin Hopper}

${ }^{T}$ UCL Eastman Dental Institute, London, United Kingdom Session 2B: Photodynamic Therapy (PDT), May 6, 2021, 15:00 - 16:00

\section{Biography:}

I have been involved in clinical PDT for the last 30 years at UCL and undertaken early phase studies on Photofrin, ALA and Foscan.
Head and neck cancer is a morbid condition with little change in survival over the last 50 years. The condition is a locoregional one and therapy often involves major ablative surgery. PDT has been shown to be an effective treatment with good functional and aesthetic outcomes. However, despite many peer-reviewed publications, it has not found widespread applications in the United Kingdom.

In this presentation, I will update the global state of PDT and describe an innovative PDT project in India where treatment resources are limited but particularly oral cancer is a major health burden.

Finally, I will examine the ongoing studies on newer therapies and look to a future where PDT will establish itself in mainstream treatment.

O08 Carbon dioxide laser and photodynamic therapy for the management of basal cell carcinomas

Miss Parneet Gill ${ }^{1}$, Miss Charlotte Defty ${ }^{1}$

${ }^{T}$ St Helens And Knowsley Trust, Liverpool, UK

Session 2B: Photodynamic Therapy (PDT), May 6, 2021, 15:00 - 16:00

\section{Biography:}

Parneet Gill is a locum plastic surgery consultant at St Helens and Knowsley NHS trust with a specialist interest in $\mathrm{CO}_{2}$ laser PDT treatment of BCCs, laser ablation of rhinophyma and laser treatment of vascular malformations.

Introduction: Basal cell carcinomas (BCCs) commonly present to the plastic surgeon; however some patients may not be suitable surgical candidates or may choose not to opt for surgery. We propose management of these patients using a combination of $\mathrm{CO}_{2}$ laser and PDT.

Method: The BCC is marked, and local anaesthetic is administered. A $\mathrm{CO}_{2}$ ablative laser is used to accurately target the area marked. Metvix is applied to the treatment area and covered for 3 hours, followed by photodynamic light activation using an Aktilite for 9 minutes. Chloramphenicol ointment is used until the second procedure 2 weeks later. The patient is reviewed at 4 weeks, 3 months and 6 months in OPD. Results: We have treated 91 patients with a total of 96 BCCs from 2017 to 2021 in a single plastic surgery laser unit. The follow up period was 4 years $(0-4)$. There were 2 recurrences, 1 case could not tolerate the second course of treatment and 1 case had an additional third course of treatment for a very large BCC.

The patient satisfaction surveys showed minimal pain scores and overall, the patients were very satisfied with the $\mathrm{CO}_{2}$ laser PDT.

Conclusion: $\mathrm{CO}_{2}$ laser PDT treatment is an additional tool when managing complex patients presenting with BCCs, who may be unsuitable for surgery or refuse to undergo it. The goal of treatment is tumour control, ideally cure if possible, but the main advantage is there is always an option of re-treating.

\section{INV09 How to buy a laser or EBD}

\section{Dr Martin Kassir ${ }^{1}$}

${ }^{7}$ Worldwide Laser Institute, United States of America Session 3A: Skin Lasers, May 7, 2021, 13:00 - 14:00

\section{Biography:}

Dr Martin Kassir is a Board-Certified Dermatologist specialising in aesthetic dermatology and laser procedures and has practiced dermatology since 2000. An internationally recognized speaker and educator on aesthetic dermatology procedures, he has lectured and conducted dermatology seminars on six continents and served as Course Instructor on Lasers and IPL, DermaFrac, Botox, Dysport, Fillers, Microneedling and Radiofrequency Devices. Dr. Kassir is Director of the Worldwide Laser Institute, a center for education and training. 
As the number of lasers and Energy Based Devices grow, so does the confusion about their utility, initial cost, and consumables.

Which device do I buy? Do I begin with one device? Which one should I buy first? How will this device effect my practice?

In this lecture, Dr. Martin Kassir will review his checklist for How to Buy a Laser. Each practitioner has their own special patient base, area of need, and area of interest. Understanding Laser Physics is a foundation for buying great devices for each practice. Then you apply other factors unique to your practice to decide which device or devices will best serve your patient population.

O09 Pulsed dye laser: a new case of successful treatment for reactive angioendotheliomatosis

Dr Kalina Bridgewater ${ }^{1}$, Dr Victoria Vilenchik ${ }^{1}$, Ms Deanna $\mathrm{Ngo}^{1}$, Dr Beth Wright $^{1}$, Dr Jon Oxley ${ }^{1}$, Dr Georgios Kravvas ${ }^{1}$, Ms Emma Hitchens $^{1}$, Dr Kay Thomas ${ }^{1}$, Dr Emma Ormerod ${ }^{1}$, Dr Daniel Keith ${ }^{1}$

${ }^{I}$ North Bristol NHS Trust, Bristol, United Kingdom

Session 3A: Skin Lasers, May 7, 2021, 13:00 - 14:00

\section{Biography:}

Dr Kalina Bridgewater MBBS, BSC

Clinical Fellow in Dermatology and Medical Education

North Bristol NHS Trust

Background and Objective: Reactive angioendotheliomatosis (RAE) is a rare cutaneous proliferative disorder of endothelial cells which can result in painful and disfiguring ulcerative lesions of the skin. There is no current proven treatment for this condition. We present a 38 year-old gentleman with extensive necrotic abdominal ulceration secondary to RAE. Pulsed dye laser (PDL) treatment has been reported in the treatment of Kaposi's sarcoma, which histologically mimics RAE. We therefore hypothesised that PDL could be effective in our gentleman's case.

Methods: The Cynosure Cynergy ${ }^{\mathrm{TM}}$ pulsed dye laser of wavelength 585 nanometres was used to deliver two treatments to the patient, seven weeks apart. The open ulcerated areas of his abdominal lesion were treated using a 10 millimetre spot size, a pulse width of 0.5 milliseconds and 6.0 Joules/ $\mathrm{cm}^{2}$ fluence.

Results: Following PDL treatment, there was a dramatic improvement in wound healing, with over $50 \%$ resolution of the ulcerated areas. Moreover, there was a significant reduction in pain and bleeding associated with the wound. Prior to treatment, these symptoms had resulted in the use of a wheelchair; however following laser therapy the patient was able to mobilise independently. No side effects of laser treatment were reported.

Conclusion: We present a case of successful and safe PDL treatment of RAE, demonstrating an improvement in both wound healing and the patient's quality of life. We suggest that PDL should be further evaluated as a treatment for RAE.

O10 Laser tattoo removal using R20M ${ }^{\mathrm{TM}}$ method. Pilot study results

\section{Ms Magdalena Atta-Motte ${ }^{1}$}

${ }^{T}$ Certifa Aesthetics Training Academy, London, United Kingdom

Session 3A: Skin Lasers, May 7, 2021, 13:00 - 14:00

\section{Biography:}

Founder of Certifa Aesthetics Training Acadmy. Author of over 20 papers published. Visiting lecturer and international trainer on lasers and EBD. Member of American Society for Laser Medicine and Surgery (ASLMS), BMLA (British Medical Laser Association).

It has been estimated that up to a quarter of young to middle-aged adults have at least one tattoo. As more tattoos are being acquired, increasing numbers of people are also seeking their removal.
Unfortunately, laser tattoo removal is generally more costly, more painful, and takes more time and more patience to have it done.

Tattoos are created by the introduction of exogenous pigment into the dermal skin layer and can be divided into professional, amateur, cosmetic, traumatic, or medical tattoos.

The aim of this presentation is to demonstrate how a unique protocol of R20M TM method can be used successfully in removing tattoos, in an effective disciplined timing and cost wise modalities.

In the described method R20M ${ }^{\mathrm{TM}}$, first pass is performed with Er:Yag fractional ablative laser followed by three to five subsequential passes with QS Nd:Yag (Xlase Plus, Biotec Italia). The Fiflow® mixture of perfluorocarbons is used to enhance the results and to reduce the effect of skin frosting by laser-assisted drug delivery (LADD) technique.

10 participants were involved in this pilot study with various types and location of the tattoos.

The $\mathrm{R} 20 \mathrm{M}^{\mathrm{TM}}$ technique is a very effective tool in a single treatment session than the conventional multiple laser sessions in tattoo removal. This method does not need any newer laser technology, clears the tattoo fast, and cuts down unnecessary treatment duration and cost. In 4 of 6 participants, cosmetic tattoo was satisfyingly eliminated; professional tattoo was cleared in 2 of 4 participants after a single session.

INV11 Photoantimicrobials: all mouth?

Prof. Mark Wainwright ${ }^{\mathbf{1}}$

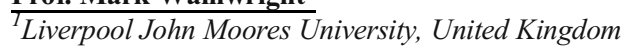

Session 4A: Lasers in Dentistry (Oral Surgery, Implantology and Endodontics), May 7, 2021, 13:00 - 14:00

\section{Biography:}

Prof. Mark Wainwright has been in photosensitiser R\&D since 1987, initially in the photodynamic therapy of cancer, but for almost 30 years in infection control applications, especially concerning conventional drug resistance. He formed the spin-out drug-discovery company Pharmalucia in 2008. His book Photosensitisers in Biomedicine was published in 2009. Mark has been Professor of Antimicrobial Chemotherapy at LJMU since 2011.

The use of light-activated antimicrobials, or photoantimicrobials, allows rapid microbial kill regardless of conventional drug resistance profile in the target, and without the potential for resistance development. From a photoantimicrobial perspective the oral cavity can offer relatively simple targets in terms of infectious disease presentation. However, the requirement for targeted light delivery and the presence of in situ oxygen might be seen as problematic, for example, in deep, anoxic infected milieux.

Similarly, the propensity of photoantimicrobials to aggregate might decrease killing potential. Such situations are considered here and ways forward suggested.

The use of photoantimicrobials in substitution or prophylaxis are also areas of interest, both from the angle of conventional antimicrobial conservation and that of epidemic/pandemic prevention. For example, tonsillitis can be attacked directly using the photoantimicrobial methylene blue, rather than a valuable conventional antibacterial, such as ampicillin; similarly, direct lesional application will inactivate the herpes viruses involved in cold sores around the mouth. From a prophylactic viewpoint, application of methylene blue and light to the anterior nares will also kill bacterial causes of meningitis and inactivate coronavirus. Such decolonisation work has been carried out for MRSA in elective patients for several years, with significant effects on post-operative infections. Additionally, since photoantimicrobials offer a properly antimicrobial approach fungal infection such as oral candidiasis can also be treated. This includes the currently problematic Candida auris.

Methylene blue is an inexpensive, efficient, non-toxic photoantimicrobial. Red light sources are plentiful. Oxygen is free! What's stopping us? 
O17 Laser-assisted treatment in management of adult ankyloglossia

Dr Dix Premsingh Prasath, Dr Reem Hanna

${ }^{7}$ Lovesmile - Dental Implants \& Laser Dentistry, Liverpool, United Kingdom

Session 4A: Lasers in Dentistry (Oral Surgery, Implantology and Endodontics), May 7, 2021, 13:00 - 14:00

\section{Biography:}

Dr Dix Premsingh Prasath graduated in 2003 in India, moved to the UK in 2005, pursued Masters degree in Restorative Aesthetic Dentistry from Manchester University and Implant Dentistry from University of Warwick. He belongs to the first batch of dentists to do the fellowship from Eastman Dental Institute, UK.

Background and Objectives: Lingual frenectomy is a surgical approach in the management of ankyloglossia (tongue-tie). The conventional surgical approach has been the gold standard of tongue-tie management. However, utilization of surgical diode laser $940 \mathrm{~nm}$ and Er,Cr:YSGG has captured the interest of many clinicians in daily practice. Diode $940 \mathrm{~nm}$ has a great affinity to haemoglobin and melanin pigmentation, whereas Er,Cr:YSGG has a great affinity to water.

This clinical case series performed to demonstrate the efficacy of utilizing two wavelengths at a therapeutic power output in the management of ankyloglossia with minimal post-operative complications and patients' acceptance of treatment.

Case Description: The patients were $>18$-year-old presented with a degree of ankyloglossia, causing a great impact in maintaining a good oral hygiene and an impairment in speech. Laser-assisted treatment: Er, Cr:YSGG (2780 nm): utilised to break down the lingual attachments at a power output of $1.5 \mathrm{~W}$, frequency: $30 \mathrm{~Hz}$, contact mode, with $40 \%$ water and $20 \%$ air, as a cooling method. Surgical diode laser $940 \mathrm{~nm}$ utilised to achieve haemostasis at a low power setting of $0.9 \mathrm{~W}$, in a pulsed mode (100 microseconds) in a contact mode.

Results: No peri- or immediate laser treatment complications reported. Additionally, the patients reported no early or late post-treatment complications and uneventful healing achieved with no relapse on the follow-up appointment.

Conclusion: Laser therapy is a non-invasive and acceptable treatment modality in management of ankyloglossia with no post-operative complications and maintains the integrity of the vital structures.

O18 Versatility of $810 \mathrm{~nm}$ diode laser in the management of oral soft tissue lesions

Dr Snehal Dalvi ${ }^{1}$, Prof Stefano Benedicenti ${ }^{2}$, Prof Reem Hanna ${ }^{2,3}$ ${ }^{T}$ Department of Periodontology, SDKS Dental College and Hospital, Nagpur, India, ${ }^{2}$ Department of Surgical Sciences and Integrated Diagnostics, Laser Therapy Centre, University of Genoa, Genoa, Italy, ${ }^{3}$ Department of Oral Surgery, King's College Hospital NHS Foundation Trust, London, United Kingdom

Session 4A: Lasers in Dentistry (Oral Surgery, Implantology and Endodontics), May 7, 2021, 13:00 - 14:00

\section{Biography:}

She has completed her MDS (Periodontology) from India. She has obtained Fellowship in Laser Dentistry [University of Genoa, Italy (UniGe)] from UCL-Eastman Dental Institute, UK and MSc (Laser Dentistry) from UniGe, Italy. Currently, she is a Senior Lecturer in the Department of Periodontology, SDKS Dental College and Hospital, Nagpur, India.

Background and Objectives: The photonic energy of surgical diode lasers is predominantly absorbed by haemoglobin in oral soft tissues and poorly absorbed by hydroxyapatite in teeth and bone. Hence, this group of lasers are suitable for soft tissue ablation as a safer procedure with lower risk of damage to hard tissue underneath the targeted soft tissues. The present clinical case series was performed to demonstrate the efficacy of $810 \mathrm{~nm}$ diode laser at therapeutic power setting in the management of various oral soft tissue lesions.

Case description: Ten patients aged 16-40 years old presented with the following oral soft tissue lesions: aberrant frenal attachment, dark pigmented gingivae, bilateral operculum associated with partially erupted mandibular third molars, plaque induced gingival enlargement and traumatic fibroma. Materials and Methods: The patients underwent $810 \mathrm{~nm}$ diode laser assisted surgical management for the same. Each surgical treatment site was lased using an initiated fibre in contact with the target tissue using the following protocol: maximum average power- $1.5 \mathrm{~W}$, continuous emission mode, beam diameter- $400 \mu \mathrm{m}$, spot area at tissue- $0.0013 \mathrm{~cm}^{2}$, maximum fluence-200 J/ $\mathrm{cm}^{2}$, maximum irradiance- $1300 \mathrm{~W} / \mathrm{cm}^{2}$, maximum total energy-500 J, maximum total treatment duration-300 seconds.

Results: The patients did not report any immediate or delayed post-operative complications and demonstrated favourable healing outcomes up to 3 months.

Conclusion: Utilization of $810 \mathrm{~nm}$ diode laser for surgical management of oral soft tissue lesions can be considered safe, effective and justifiable over the conventional scalpel technique though it must be performed by trained and experienced clinicians only.

INV10 Using fractional lasers in Indian patients for post acne scars

\section{Professor Koushik Lahiri ${ }^{1}$}

${ }^{7}$ Apollo Gleneagles Hospitals And Wizderm, India Session 3B: Skin Lasers, May 7, 2021, 14:15 - 15:15

\section{Biography:}

A Professor and Senior Consultant Dermatologist of Apollo Gleneagles Hospital in Kolkata, India, and the Honorary Director of WIZDERM. Also VP of the International Society of Dermatology, past Secretary General of IADVL and past President of ACSI, Fellow of IADVL Academy of Dermatology, International Fellow of AAD, Foundation Fellow of Asian Academy of Dermatology and Venereology and member of EADV. Also a Fellow of all three Royal Colleges of England; London, Glasgow and Edinburgh.

Most common scar conditions dermatosurgeons treat in their practice are acne scars, hypertrophic scars, keloids and burn scars. With wider availability of lasers and energy-based devices, many conditions where quality, alignment or proportion of collagen, elastin in tissue is disturbed and where selective dermal ablation leading to its renewal may alter the pathology are treated using various kinds of lasers. Thickness or depth, contour, vascularity and pigment are key parameters targeted in laser scar reduction. Lasers have a role in all the phases of life cycle of scar from formation till recurrence. Deep fractional $\mathrm{CO}_{2}$ lasers have evolved as superior and safer option. While generalized collagen tightening of scar affected skin is achieved by most fractional devices, scar shouldering is required for deeper scars like icepick scars and boxcar scars. Lasers are one of the adjuvant therapies for scars and must be combined with established therapies like pressure, intralesional injections, subcision for superior results.

O11 Rhinophyma - from the genetics of the disease to our therapeutic protocol

\section{Mrs Maria Roxana Chiru ${ }^{1}$}

${ }^{7}$ Bedford Hospital NHS Trust, Bedford, United Kingdom

Session 3B: Skin Lasers, May 7, 2021, 14:15 - 15:15

\section{Biography:}

Roxana Chiru, MD, PhD, BTEC (LASER), FRCS (ROPLAST)

Consultant Plastic, Reconstructive and Laser Surgeon

Plastic Surgery and Laser Centre Bedford Hospital 
Rhinophyma is a chronic and progressive condition considered a form of severe rosacea that classically affects the tip of the nose. Its pathogenesis is not fully elucidated, thus making it difficult to prevent and cure. From what we know so far, rhinophyma is a multifactorial disease. Research into the genetics, histopathology and mechanisms of this condition is showing a genetic component with changes at the level of glutathione S-transferases and HLS complex resulting in increased inflammation and decreased protection from the oxidative stress. Altered immune response has been suggested by increased number of mast cells in the affected areas, overexpression of TGF $\beta 2$ and FXIIIc. There is also an androgenic component suggested by the male predominance. These translate in disruption of the skin barrier with telangiectatic growth, chronic inflammation and oedema, sebaceous glands and connective tissue hypertrophy, fibrosis and a risk of 15-30\% malignant transformation.

A proper treatment needs to assess all these aspects. There are a range of therapies from topical medication to surgical procedures and lasers, with the laser being the preferred treatment in terms of risks/benefits ratio.

We propose a new protocol for the laser treatment of rhinophyma, combining the ablative and vascular lasers, including yearly follow-ups post ablation and top ups with the vascular lasers for a better control of the condition and a longer disease-free interval.

O12 Combined ablative $\mathrm{CO}_{2}$ laser and triamcinolone acetonide injections for auricular keloid scars: a case series

Dr Dina Ismail ${ }^{1}$, Dr Vishal Madan ${ }^{1,2}$

${ }^{T}$ Laser Division, Department of Dermatology, Salford Royal NHS Foundation Trust, Salford, UK, ${ }^{2}$ Everything Skin Clinic, Haw Bank House, Cheadle, UK

Session 3B: Skin Lasers, May 7, 2021, 14:15 - 15:15

\section{Biography:}

Dr Ismail is a Dermatology Specialist Registrar, based in the North West Deanery, with an interest in Lasers.

Introduction: Keloid scars occur due to an abnormal tissue response to dermal injury. They are cosmetically undesirable and cause symptoms including pain, burning and pruritus. Regression is rare without treatment; however this can be challenging and recurrences are common.

Methods: A retrospective case note review of 15 patients with auricular keloids presenting to a NHS tertiary laser center between 2012 and 2018, and a private dermatology clinic, was performed. We assessed the presentation, management and outcomes of treatment of auricular keloids with carbon dioxide $\left(\mathrm{CO}_{2}\right)$ laser and intralesional triamcinolone acetonide in these patients.

Results: All 15 patients presented with auricular keloid scars secondary to ear piercing. The mean age at presentation was 22 years (range 15-31 years). Half had previously undergone failed treatment with repeat triamcinolone injections and/or surgical excision. All patients were treated with ablative carbon dioxide $\left(\mathrm{CO}_{2}\right)$ laser, used in cutting and resurfacing mode, with adjunctive intralesional triamcinolone acetonide. Fourteen patients required only 1 laser treatment. The number of intralesional steroid injections required ranged from 1 to 6 injections at regular intervals. Four patients were lost to follow-up and 11 patients reported good treatment outcome with no recurrence at follow up (range 6 weeks to 18 months). Two patients with post laser erythema were treated with pulsed dye laser and a further patient required oral antibiotics for a laser treatment associated infection.

Conclusion: Dual $\mathrm{CO}_{2}$ laser ablation and intralesional steroid treatment is a useful treatment strategy for auricular keloid scars, particularly in patients who have failed repeated steroid injections or surgical excision.
INV12 Photobiomodulation in the management of side effects of cancer treatments: rationale, mechanisms of action, indications and clinical protocols

\section{Professor René-Jean Bensadoun}

Session 4B: Low Level Lasers (LLLT) and LEDs in Medicine and Dentistry, May 7, 2021, 14:15 - 15:15

\section{Biography:}

Professor in Radiation Oncology, Chairman, Centre de Haute Energie, NICE (France), Chairman, WALT 2018 World Congress, WALT President (2021-24).

2020 WALT Senior Award for Excellence in Photobiomodulation Clinical Sciences

Fields of Interest: Photobiomodulation, Radiation Oncology, Head \& Neck Cancer, Supportive Care in Cancer, New techniques in Radiation Oncology (IMRT, stereotaxy), Cancer-treatment toxicity. Permanent en Oncologie-Hématol (CPOH) de l'ANSM (since 2019). Deputy Associate Editor JSCC (Supp Care in Cancer). Member MASCC-ISOO Mucositis \& Oral Care Boards, and of ESMO Toxicity Working Group. DU PBM in Oncology (IGR, Fr).

Purpose: There is a large body of evidence supporting the efficacy of low-level laser therapy (LLLT) also known as photobiomodulation (PBM) when used for the prevention and/or treatment of oral mucositis $(\mathrm{OM})$ in patients undergoing radiotherapy for head and neck cancer (HNC). Recent advances in LLLT/PBM technology, together with a better understanding of mechanisms involved and dosimetric parameters, may lead to the management of a broader range of complications associated with cancer treatment, dermatitis being one of them.

Results: In vitro studies assessing the effect of LLLT/PBM on tumor cells have reported conflicting results. This diversity of effects is likely to be specific to LLLT power and dose. However, no clinical studies reported tumor protection or enhanced tumor growth as a result of LLLT exposure. For OM management, optimal LLLT/PBM parameters were: Wavelength: typically between $633-685$ nanometer $(\mathrm{nm})$, or $780-830$ $\mathrm{nm}$; Energy density: laser or light-emitting diode (LED) output between 10-150 milliwatts; Dose: 2-3 Joules $\left(\mathrm{J} / \mathrm{cm}^{2}\right)$, and no more than $6 \mathrm{~J} / \mathrm{cm}_{2}$ on the tissue surface treated; Schedule: $2-3$ times a week up to daily; Emission type: continuous or pulsed $(<100 \mathrm{Hertz})$ as low frequency pulsed light may be superior to continuous wave light for wound healing; Route of delivery: intra-orally or transcutaneously.

Although evidence suggests that LLLT/PBM is safe in HNC patients, vigilance remains warranted to detect any potential adverse effects of LLLT/PBM on cancer treatment outcomes and survival.

O19 Role of light therapy in COVID-19 management: rationale and mechanism of action

Dr. Reem Hanna ${ }^{1,3}$, Dr Snehal Dalvi ${ }^{2}$, Professor Stefano Benedicenti ${ }^{3}$ ${ }^{T}$ Department of Oral Surgery, King's College Hospital NHS Foundation Trust, London, United Kingdom, ${ }^{2}$ Department of Periodontology, SDKS Dental College, Nagpur Maharashtra, India, ${ }^{3}$ Department of Surgical Sciences and Integrated Diagnostics, University of Genoa, Italy, Genoa, Italy

Session 4B: Low Level Lasers (LLLT) and LEDs in Medicine and Dentistry, May 7, 2021, 14:15 - 15:15

\section{Biography:}

Dr. Reem Hanna BDS, MSc Oral Surgery (UCL/London), MSc laser dentistry (UNIGE), PGDipSed (KCL/London) PGCAP (KCL/London), PGDipAC (KCL/London) FAHE.

- Associate Specialist in Oral Surgery, King's College Hospital NHS

Foundation Trust. 
- Registered Specialist Oral Surgeon in UK.

- Honorary Senior Lecturer at UCL/ Eastman Dental Institute/ London/ $U K$

- Professor a.c., at the Department of Surgical Sciences and Integrated Diagnostics, University of Genoa, Italy.

- Course leader of the fellowship course in laser dentistry. UCL/Eastman Dental Institute.

- Currently a PhD student.

- Reem is also heavily involved in research in utilising laser therapy in neuropathic pain, tissue regeneration and surgery.

Phototherapy (PDT and photobiomodulation therapy) is a non-invasive vehicle, which can significantly contribute to mitigate COVID-19 pandemic. The photonic energy of photodynamic therapy (PDT) can inactivate SARS-CoV-2 virus, whereas photobiomodulation therapy (PBM therapy) can regulate COVID-19 cytokines storm.

Since SARS-CoV-2 virus first starts in the upper respiratory airways (URA), particularly the front of the nose, so methylene blue-inducing PDT, as a non-toxic dye activated by suitable light source when applied in the nostril can destroy the virus before it spreads into the lower respiratory tract (lungs). This treatment targets the virus at the early stages of infection when the virus is mostly confined to URA and not yet spread to the lungs. While PBM therapy, as a single or an adjunctive therapy to standard care can potentially regulate the cytokines storm generated by the virus, leading to reduce the disease progression, minimising hospitalisation and the need for ventilation in COVID-19 patients.

This talk aims to provide an overview of rationale, evidence-based science and the current clinical trials, in utilising phototherapy in COVID-19 management.

O20 2021 Industry review for cold laser therapy

\section{Ms Ruth Phypers ${ }^{1}$}

${ }^{T}$ Laser Medicine, London, United Kingdom

Session 4B: Low Level Lasers (LLLT) and LEDs in Medicine and Dentistry, May 7, 2021, 14:15 - 15:15

\section{Biography:}

Ruth Phypers MA (SOAS, University of London) builds on her experience in leading-edge technology since 1992, combining extensive therapeutic expertise with Low Level Laser (LLLT) to offer a general health practice in Photobiomodulation. Based at 10 Harley Street, Laser Medicine utilises LLLT protocols developed over 40 years.

Laser Therapy has become more widely available over the last few years thanks to the number of trained practitioners in the field that employ the use of Class $3 \mathrm{~b}$ Laser for healing across a wide range medical and healthcare practices. Cold Laser Therapy, also known as Low Level Laser Therapy (LLLT), has gained wide appeal for the treatment of pain, inflammation, tissue repair, sports injury, etc., and many osteopaths, physiotherapists, podiatrists and chiropractors integrate the use of Cold Laser into their practice. Cold Laser Therapy has also enjoyed recent growth in the aesthetics industry, and many skin specialists embrace its natural healing effect to increase the production of collagen, without creating a thermal or ablative reaction in the skin tissue.

On a wider scale, Cold Laser Therapy produces positive results for the treatment of other health conditions, with clinical research indicating the effectiveness of Laser Therapy for post-chemotherapy recovery, fertility, hypothyroidism, Hashimoto's Disease, enlarged prostate (BPH), Parkinson's, Alzheimer's, and stroke recovery to name but a few.

This paper aims to provide a current view of the Cold Laser Therapy/Low Level Laser Therapy (LLLT) field including current research from 2020, including for symptomatic recovery of COVID-19. I address some of the anomalies, inconsistencies and legitimate areas of confusion that I have identified in both the general health and aesthetics fields. In conclusion I look to the future of Cold Laser Therapy, identifying what I believe to be the strongest and most exciting areas of growth.

O13 Laser or no laser therapy for multiple glomuvenous malformations

Dr Giulia Rinaldi ${ }^{1}$, Dr Samira Batul Syed ${ }^{2}$

${ }^{T}$ Guy's and St Thomas' Hospital, United Kingdom, ${ }^{2}$ Great Ormond Street Hospital, United Kingdom

Session 3C: Skin Lasers, May 7, 2021, 15:30 - 16:30

\section{Biography:}

Dr Giulia Rinaldi is an academic foundation doctor at Guy's \& St Thomas' Hospital.

Introduction: Glomuvenous malformations (GVM) are rare, benign vascular anomalies characterised by cutaneous lesions consisting of distended vascular channels with abnormal glomus cells. GVMs are progressive increasing in size and darkening in colour with age. Furthermore, they often cause patients severe pain. Treatment for GVMs has often been excisional surgery. Laser therapy has recently emerged as a treatment method to delay the progression of the lesions and avoid multiple surgical excisions.

Methods: All patients who presented with multiple GVMs to the GOSH dermatology department over the last 10 years were reviewed. Clinical outcomes of patients who underwent laser therapy for their GVMs compared to those that did not undergo any laser therapy were compared. Outcomes included clinical photograph review and quantification of surgical excision therapies performed.

Results: Six patients presented with multiple GVMs over the last 10 years. Three of these patients underwent multiple laser sessions, whilst three did not undergo any laser sessions. The three patients that underwent laser treatment had marked improvement in clinical appearance and reduction in symptoms. None of these patients required any surgical excision. The three patients who did not undergo laser treatment experienced marked clinical progression of their GVMs. These all underwent surgical procedures. Laser therapy was well tolerated and there were no reported side effects. Conclusion: Laser therapy for GVMs is a safe and successful method to obtain good cosmetic and clinical outcomes. All our patients that received laser therapy did not need to undergo surgical removal of the GVM lesions.

O14 Efficacy of lasers and light-based devices in long-term hair-reduction defined by body site-specific growth-cycles

Dr Sanjaykumar Rajpara ${ }^{2,3,4}$, Miss Albana Krasniqi ${ }^{1}$

${ }^{1}$ University Of Aberdeen, Aberdeen, Scotland, '2Grandaura Skin and Wellbeing Clinic, Aberdeen, Scotland, ${ }^{3}$ Harley Street Dermatology Clinic, London, United Kingdom, ${ }^{4}$ Sk:n London, London, United Kingdom

Session 3C: Skin Lasers, May 7, 2021, 15:30 - 16:30

\section{Biography:}

Intercalating medical student at the University of Aberdeen with an interest in Dermatology.

Background and Objectives: Laser and light-based devices provide scope for "long-term hair-removal"; however, there is limited data on their long-term efficacy. Hair grows at different rates depending on body-site; hence when conducting research on "long-term" hair-reduction, it is, theoretically, important that length of hair-growth cycles is taken into account and adjusted for. This study aimed to assess the long-term efficacy of laser and light-based "hair-removal" devices, taking into account body site-specific growth-cycles. 
Methods: A systematic review of randomised controlled trials (RCTs) with follow-up periods greater than or equal-to the length of one complete hair growth-cycle in the body-site targeted was conducted. Only five eligible RCTs were identified as suitable for inclusion and these comprised a total of 223 patients.

Results: The average long-term hair-reduction reported for neodymium: yttrium-aluminium-garnet laser ranged from 30 to $73.61 \%$, Alexandrite laser ranged from 35 to $84.25 \%$ and Diode laser from 32.5 to $69.2 \%$. In all three devices, the greatest long-term reduction was observed from trials targeting leg-hair (1-year growth-cycle) and lowest from targeting facial-hair (6-month growth-cycle). Intense pulsed light produced average long-term hair-reduction of 52.7-27\%; smallest reduction was observed in targeting the face-area and greatest from targeting the axillary-area (7-month growth-cycle).

Conclusion: While the superiority of one device over another in long-term hair-reduction was not demonstrated, a trend towards greater long-term hair-reduction on body-sites with longer hair growth-cycles was observed. Therefore, it is important that follow-up periods in future trials assessing hair-reduction interventions take into account hair growth-cycles at individual body-sites. This may provide more accurate long-term data on patient-relevant treatment outcomes.

$\mathrm{O} 15 \mathrm{CO}_{2}$ Laser treatment of keratinocytic epidermal naevi: a case series

Dr Dina Ismail ${ }^{1}$, Dr Vishal Madan ${ }^{1}$

${ }^{T}$ Laser Division, Department of Dermatology, Salford Royal NHS

Foundation Trust, Salford, UK

Session 3C: Skin Lasers, May 7, 2021, 15:30 - 16:30

\section{Biography:}

Dr Ismail is a Dermatology Specialist Registrar in the North West Deanery, with an interest in Lasers.

Introduction: Epidermal naevi (EN) are benign hamartomatous growths, mostly presenting at birth or during the first year of life. Keratinocytic naevi are the most common subtype and usually appear as linear or whorled papilliform plaques along the lines of Blaschko. Although benign, the cosmetic appearance of EN can impact quality of life and therefore treatment is frequently sought. Options range from topical therapies, which are less effective, to fully ablative carbon dioxide $\left(\mathrm{CO}_{2}\right)$ laser.

Methods: A retrospective case review of presentation, treatment and outcomes was performed in 23 patients presenting with keratinocytic EN to a tertiary NHS laser center.

Results: The mean age at presentation was 16 years (range 5-56 years). Most patients $(n=12)$ presented with cosmetically obvious EN affecting the head and neck region, other areas were the trunk and arms. All 23 patients underwent ablative $\mathrm{CO}_{2}$ laser treatment and of these; 2 had previously undergone surgical excision/debridement. A good cosmetic outcome was achieved in 19 patients. The majority experienced no complications; however one patient developed residual erythema and a hypertrophic scar, treated with pulsed dye laser and triamcinolone acetonide injections, respectively. Two patients developed early recurrence at 4-month follow-up. A further patient reported pruritus at the laser treatment site and was treated with triamcinolone acetonide injections.

Conclusion: The $\mathrm{CO}_{2}$ laser is an effective treatment modality in adult and paediatric patients presenting with keratinocytic EN and can obtain a good cosmetic outcome with minimal side effects.

O16 Carbon dioxide laser ablation of rhinophyma.....the end of surgical excision?

Miss Parneet Gill ${ }^{1}$, Mr Maxwell Murison ${ }^{2}$, Mrs Barbara O'Leary ${ }^{2}$, Miss Charlotte Defty

${ }^{1}$ St Helens And Knowsley NHS Trust, Liverpool, UK, ${ }^{2}$ Morriston Hospital, Swansea, Wales

Session 3C: Skin Lasers, May 7, 2021, 15:30 - 16:30
Biography:

Parneet Gill is a locum plastic surgery consultant in St Helens and Knowsley NHS trust with a specialist interest in laser $\mathrm{CO}_{2} \mathrm{PDT}$ treatment of BCCs, laser ablation of rhinophyma and treatment of vascular malformations.

Introduction: The management of rhinophyma with surgical excision may include over-excision, extensive scarring, asymmetry, risks of general anaesthesia and inpatient treatment cost. Carbon dioxide $\left(\mathrm{CO}_{2}\right)$ laser ablation enables a single stage procedure under local anaesthetic (LA) as a daycase, with minimal blood loss and scarring, and superior shaping of the nose.

Methods: We present our experience of $\mathrm{CO}_{2}$ laser ablation under LA. All patients were consented regarding risks of laser ablation and anticoagulants were continued. Postoperative instructions included sun protection and regular application of topical chloramphenicol ointment or Vaseline. Patients were followed up at 1-3 weeks and 36 months.

Results: 38 patients were treated by 2 specialist laser consultants between May 2017 and February 2021, with a technique mirrored from the plastic surgery laser unit at Swansea Hospital. The rhinophymatous changes ranged from mild to severe, some with severe impact on the quality of life of patients, from a cosmetic and functional point of view. Difficult cases, including those who had previously undergone surgical excision, were successfully managed with improved scarring. One case was re-treated, one developed poor scarring from facemask sticking to wound and one case recurred due to aggressive rosacea. No postoperative bleeding or infections occurred, and high patient satisfaction was noted.

Conclusion: Rhinophyma can be successfully managed in a single stage, under LA, using $\mathrm{CO}_{2}$ laser ablation in the daycase setting. It avoids the complications associated with surgical excision and general anaesthesia and has a high rate of patient satisfaction, with excellent cosmetic and functional outcomes.

INV14 Lasers in the management of filler complications

\section{Dr Firas Al-Niaimi}

Session 5: New and Developing Treatments, May 7, 2021, 15:30 - 16:30

\section{Biography:}

Dr Firas Al-Niaimi is a highly experienced consultant dermatologist, dermatologic surgeon and laser expert with over 20 years' experience and an international standing and trained in some of the UK's top hospitals and currently holds an honorary consultant position at the world-renowned St. John's Institute of Dermatology at Guy's and St. Thomas' Hospital where he completed an advanced fellowship in dermatologic surgery and skin cancers.

He has a passion for education, research and science and has over 150 publications in Dermatology and lasers making him one of the most prolific and well-published consultant dermatologists in the UK. In addition to this he has more than 200 scientific presentations all around the world where he is regularly invited to speak.

He works at the renowned London Scar Clinic and has particular expertise and interest in the use of lasers in scar management.

Fillers are widely used in cosmetic practice and while most of the complications are transient nevertheless some can be debilitating and may require specialist intervention. Lasers have been used in a number of filler complications presentations with very good outcome and its use in this field may grow given the rise in the popularity of filler use.

Lasers have been successfully used in the management of bruising post filler as well as Tyndall effect due to the superficial placement of hyaluronic acid filler. The use of a fibre-optic intralesional laser in filler nodules and sclerotic subtype granulomas of fillers is 
relatively new and has been reported with high degree of success. Laser practitioners should be aware of the increasing role of lasers in the management of some filler complications.

O21 Laser treatment of epistaxis in hereditary haemorrhagic telangiectasia patients and the effect of withholding treatment

Dr. Mark Brewin ${ }^{1}$, Simon Dennis ${ }^{1}$, Yuchen Jiang ${ }^{1}$

${ }^{7}$ Salisbury NHS Foundation Trust, Salisbury, United Kingdom

Session 5: New and Developing Treatments, May 7, 2021, 15:30 - 16:30

\section{Biography:}

Mark is a clinical scientist with a keen interest in lasers and burns care. He has worked within the Salisbury Laser Clinic and as R\&D lead in Burns. Mark is involved in the development and improvement of the laser service; but his main research interest is in hypertrophic burns scars.

Background and Objectives: The ENT department at Salisbury NHS Foundation Trust has been treating epistaxis for Hereditary Haemorrhagic Telangiectasia (HHT) patients for a number of years. This paper presents the treatment of this condition and its effect on their Epistaxis Severity Score (ESS). It examines the impact on quality of life from the patients. COVID-19 presented an opportunity to assess the effect of withholding the treatment.

Study design and Method: This study is a retrospective review of patients treated at Salisbury Laser Clinic (SLC) from inception up to March 2021. The patients are treated using an adapted handpiece to deliver the laser treatments to all visible parts of the nares. Epistaxis Severity Scores (ESS) are reviewed periodically for each patient. A questionnaire was presented to the patients to evaluate the worth of the treatment by way of effectiveness, treatment comfort and the patient-reported experience.

Results: The study follows the progress of 34 patients and shows the control of ESS scores after a course of treatments and also once treatment was withheld during the COVID pandemic. It also presents the qualitative data from the questionnaire on the effect on quality of life.

Conclusion: The treatment of epistaxis for this cohort of HHT patients shows to be effective and have great impact on the quality of life for the patients.

\section{$\mathbf{A}$}

\begin{tabular}{llll}
\hline Al-Niaimi, Firas & INV14 & Atta-Motte, Magdalena & O10 \\
\hline B & & & \\
& & & \\
\hline Belba, Kerry & INV04 & Brewin, Mark & 021 \\
Benedicenti, Stefano & O18, O19 & Bridgewater, Kalina & O09 \\
Bensadoun, René-Jean & INV12 & & \\
\hline
\end{tabular}

C

Chiru, Maria Roxana

O03, $\mathrm{O} 11$

D

\begin{tabular}{llll}
\hline Dalvi, Snehal & O18, O19 & Dennis, Simon & O21 \\
Defty, Charlotte & O08, O16 & & \\
\hline
\end{tabular}

G

\begin{tabular}{llll}
\hline Gill, Parneet & O08, O16 & & \\
\hline H & & & \\
\hline Hanna, Reem & O17, O18, O19 & Hitchens, Emma & O09 \\
Hart, Graham & O02 & Hopper, Colin & O07 \\
Hindocha, Sandip & O03 & & \\
\hline
\end{tabular}

I

Ismail, Dina $\quad 012,015$

$\mathbf{J}$

\begin{tabular}{llll}
\hline Jiang, Yuchen & O21 & & \\
\hline K & & & \\
& & & \\
\hline Kassir, Martin & INV09 & Krasniqi, Albana & O14 \\
Keith, Daniel & O09 & Kravvas, Georgios & O09 \\
\hline
\end{tabular}

$\mathbf{L}$

\begin{tabular}{llll}
\hline Lahiri, Koushik & INV10 & & \\
\hline M & & & \\
& & & \\
\hline Madan, Vishal & 012, O15 & Morton, Colin & INV08 \\
Maheshwari, Kavish & O03 & Muggeson, Kerry & O03 \\
Marsden, Andrew & INV05 & Murison, Maxwell & O16 \\
Marza, Liliana & O01 & Murphy, Michael & O04 \\
\hline
\end{tabular}

$\mathbf{N}$

\begin{tabular}{llll}
\hline Ngo, Deanna & O09 & & \\
\hline O & & & \\
& & & \\
\hline O'Leary, Barbara & O16 & Ormerod, Emma & O09 \\
O'Mahoney, Paul & INV07 & Oxley, Jon & O09
\end{tabular}

$\mathbf{P}$

\begin{tabular}{llll}
\hline Pelling, Vincent & O05 & Premsingh Prasath, Dix & O17 \\
Phypers, Ruth & O20 & & \\
\hline
\end{tabular}

$\mathbf{R}$

\begin{tabular}{llll}
\hline Rajpara, Sanjaykumar & O14 & Rinaldi, Giulia & O13 \\
\hline
\end{tabular}


S

Simcox, Lindsey

Smith, Rhiannon

INV03

INV01

Thomas, Kay

O09

Town, Godfrey

O06
V

Vilenchik, Victoria $\quad$ O09

W

\begin{tabular}{llll}
\hline Wainwright, Mark & INV11 & Wright, Beth & O09 \\
\hline
\end{tabular}

Publisher's note Springer Nature remains neutral with regard to jurisdictional claims in published maps and institutional affiliations. 